\title{
Triptolide suppresses the proliferation and induces the apoptosis of nasopharyngeal carcinoma cells via the PI3K/Akt pathway
}

\author{
MI WANG, BO CHEN and LIANG CHAI \\ Department of Otolaryngology, The First Affiliated Hospital, College of Medicine, Zhejiang University, \\ Hangzhou, Zhejiang 310003, P.R. China
}

Received March 17, 2018; Accepted October 19, 2018

DOI: $10.3892 / \mathrm{ol} .2018 .9726$

\begin{abstract}
Nasopharyngeal carcinoma (NPC) is an endothelium-associated malignancy that is heavily influenced by Epstein Barr virus infection. Triptolide, extracted from Tripterygium wilfordii, has been proven to possess anti-inflammatory, immunosuppressive and anti-cancerous activity. Although the effect of triptolide on numerous cancer cell types has been outlined, its effect in NPC remained unclear. The present study investigated the effects and underlying mechanisms of triptolide in C666-1 and NP69 cells. It was revealed that triptolide significantly inhibited proliferation and induced apoptosis in C666-1 cells. Increased levels of cleaved-caspase-3 and apoptosis regulator BAX, decreased expression of apoptosis regulator $\mathrm{Bcl}-2$, and reduced phosphorylation of $\mathrm{RAC}-\alpha$ serine/threonine-protein kinase (Akt), were responsible for this induction of apoptosis. Notably, pretreating C666-1 cells with the phosphatidylinositol 3-kinase (PI3K)/Akt inhibitor LY294002 suggested that with increasing concentrations of LY294002, triptolide exhibited decreasing ability to suppress proliferation and induce apoptosis in these cells. In conclusion, the results demonstrated that triptolide suppressed the proliferation and induced the apoptosis of C666-1 cells in a PI3K/Akt-dependent manner and therefore, may serve as a promising therapeutic candidate for NPC.
\end{abstract}

\section{Introduction}

Nasopharyngeal carcinoma (NPC) is an endothelium-associated malignancy with a number of unique etiological and biological characteristics $(1,2)$. The neoplasm in patients with NPC originates from the superior aspect of the pharynx and extends along the soft palate. Primary etiological factors include genetics and environment, in addition to infection with Epstein Barr

Correspondence to: Dr Liang Chai, Department of Otolaryngology, The First Affiliated Hospital, College of Medicine, Zhejiang University, 79 Qingchun Road, Hangzhou, Zhejiang 310003, P.R. China E-mail: 1515087@zju.edu.cn

Key words: triptolide, apoptosis, RAC- $\alpha$ serine/threonine-protein kinase, nasopharyngeal carcinoma virus (EBV), which presents in $90 \%$ of cases of NPC (3). There is evidence to suggest that transfection of the viral latent membrane protein 1 (LMP1) gene into normal epithelial cells triggers the immortalization of rodent cells and enhances a number of prominent characteristics of carcinoma $(4,5)$. Various signaling cascades, including those of phosphatidylinositol 3-kinase/RAC- $\alpha$ serine/threonine-protein kinase (PI3K/Akt), extracellular signal-regulated kinase and Wnt/ $\beta$-catenin are broadly activated by LMPs, and subsequently impact cell proliferation and motility (4,6,7). Notably, the PI3K/Akt signaling pathway may be involved in the inhibition of apoptosis and the promotion of proliferation (8).

Triptolide, a type of epoxy diterpene monomer, is the principal bio-active component of Tripterygium wilfordii, and is widely used in Chinese herbal medicine (9). Triptolide possesses anti-inflammatory, immunosuppressive and anti-procreation activity, and has been widely used in the treatment of autoimmune diseases and the inhibition of immune rejection in patients with organ transplants $(10,11)$. In addition, triptolide induces apoptosis in a number of cancer cell lines and increases the sensitivity of tumor cells to other anticancer drugs (12-14). Such broad-spectrum anticancer activity makes triptolide an interesting research prospect. Therefore, it was hypothesized that triptolide may represent a promising therapy option for nasopharyngeal cancer by inducing apoptosis through the PI3K/Akt pathway.

\section{Materials and methods}

Reagents. Triptolide (purity, >98\%) was purchased from Guangzhou PI\&PI Biotech, Inc. (Guangzhou, China). Rabbit polyclonal anti-cleaved caspase 3 (cat. no: 9661), anti-apoptosis regulator BAX (Bax, cat. no: 2774), anti-apoptosis regulator Bcl-2 (Bcl-2, cat. no: 2872) and anti-Akt (cat. no: 9272) and mouse monoclonal anti-phospho-Akt (cat. no: 12694) antibodies were obtained from Cell Signaling Technology (Danvers, MA, USA). Rabbit polyclonal anti-GAPDH (cat. no: A00227-1) antibody was obtained from Wuhan Boster Biological Technology, Ltd., (Wuhan, China). Horseradish peroxidase (HRP) -labeled goat anti-mouse (cat. no: 115-035-003) or rabbit (cat. no: 111-035-003) antibodies were purchased from Jackson ImmunoResearch (Bar Harbor, ME, USA). MTT (Sigma-Aldrich; Merck KGaA, Darmstadt, Germany) and LY294002 (MedChemExpress, Monmouth 
Junction, NJ, USA) were dissolved in dimethyl sulfoxide (DMSO) and stored at $-20^{\circ} \mathrm{C}$, protected from the light.

Cell culture. The human EBV-positive NPC cell line C666-1, and the immortalized normal nasopharyngeal epithelial cell line NP69 were purchased from The American Type Culture Collection (Manassas, VA, USA). The C666-1 cells were cultured in RPMI 1640 medium (Gibco; Thermo Fisher Scientific, Inc., Waltham, MA, USA) supplemented with $10 \%$ fetal bovine serum (Gibco) with $1 \%$ penicillin and $1 \%$ streptomycin. The NP69 cells were cultured in Keratinocyte-SFM medium (Gibco; Thermo Fisher Scientific, Inc.) supplemented with $10 \%$ fetal bovine serum and $1 \mathrm{ng} / \mathrm{ml}$ epidermal growth factor (PeproTech, Inc., Rocky Hill, NJ, USA). All cells were cultured at $37^{\circ} \mathrm{C}$ with $5 \% \mathrm{CO}_{2}$.

Cell proliferation assays. MTT assays were performed according to the manufacturer's protocol (Sigma-Aldrich; Merck KGaA). Cells were plated at a density of $5 \times 10^{3}$ per well in a 96-well plate, and incubated in the appropriate culture medium with $12.5,25,50$ and $100 \mathrm{nM}$ triptolide. Alternatively, the plated cells were cultured in serum-free medium for $24 \mathrm{~h}$ and pretreated with 1, 10 or $50 \mu \mathrm{M} \mathrm{LY} 294002$ for $2 \mathrm{~h}$. Subsequently, the C666-1 cells were cultured in complete medium with $50 \mathrm{nM}$ triptolide or control solvent. Tetrazolium salts were added and incubated for the final $4 \mathrm{~h}$ of the experiment. A volume of $150 \mu \mathrm{l}$ DMSO was used to dissolve the formazan crystals. The absorbances were determined at $570 \mathrm{~nm}$.

Cell cycle determination. Cells with a density of $1 \times 10^{5}$ cell/well were plated in 6-well plates. The cells were incubated with $12.5,25,50$ and $100 \mathrm{nM}$ triptolide. After $24 \mathrm{~h}$ of incubation at $37^{\circ} \mathrm{C}$, cell cycle analysis was performed. Cells were fixed with cold ethanol $(70 \%)$ at $4{ }^{\circ} \mathrm{C}$ for $12 \mathrm{~h}$, incubated with $100 \mu 1$ RNase A solution (Nanjing Jiancheng Bioengineering Institute, Nanjing, China) for $30 \mathrm{~min}$ at $37^{\circ} \mathrm{C}$, and then mixed with $400 \mu \mathrm{l}$ propidium iodide (PI) solution (Nanjing Jiancheng Bioengineering Institute) for $30 \mathrm{~min}$ at $4^{\circ} \mathrm{C}$, protected from light. The labeled cells were analyzed with a FACSCalibur flow cytometer (BD Biosciences, San Jose, CA, USA) and the data were processed with FlowJo software VX (Tree Star, Inc., Ashland, OR, USA).

Apoptosis assay. Apoptosis assays were performed using an Apoptosis Detection kit (Vazyme, Piscataway, NJ, USA) according to the manufacturer's protocol. The cells treated with triptolide were collected, resuspended and incubated in $500 \mu \mathrm{l}$ binding buffer with $5 \mu \mathrm{l}$ Annexin V and $5 \mu \mathrm{l}$ PI. After 15 min of incubation at room temperature, the labeled cells were analyzed using a FACSCalibur flow cytometer and the data were processed using FlowJo software.

Western blot analysis. Cells were lysed in radioimmunoprecipitation assay buffer containing phenylmethylsulfonyl fluoride and soybean protease inhibitor (Beyotime Institute of Biotechnology, Haimen, China). Following 13,000 x g centrifugation at $4^{\circ} \mathrm{C}$ for $15 \mathrm{~min}$, the supernatants were harvested for protein quantification using a bicinchoninic acid assay kit (Beyotime Institute of Biotechnology). A total of $40 \mu \mathrm{g}$ protein/lane was loaded, and separated using a 10 or $12 \%$ polyacrylamide gel. The proteins were transferred to a PVDF membrane (EMD Millipore, Billerica, MA, USA). Following washing three times in deionized water, the membranes were blocked with $5 \%$ skim milk for $120 \mathrm{~min}$ at room temperature, incubated with the primary rabbit anti-cleaved caspase 3 antibody, anti-Bax antibody, anti-Bcl-2 antibody, anti-Akt antibody (all Cell Signaling Technology, Inc., Danvers, MA, USA) anti-GAPDH antibody (Wuhan Boster Biological Technology, Ltd.) or mouse monoclonal anti-phospho-Akt antibody (Cell Signaling Technology, Inc.) overnight at $4^{\circ} \mathrm{C}$ (dilution, 1:1,000). Following washed three times with Tris buffered saline/ $0.5 \%$ Tween-20, the members were subsequently incubated with HRP-conjugated goat anti-rabbit or anti-mouse antibodies (dilution, 1:1,000 or 1:5,000 respectively) at room temperature for $60 \mathrm{~min}$. The membranes were visualized with an enhanced chemiluminescence kit (EMD Millipore), with GAPDH as a control. All the images were quantified by Image J 1.1 software (National Institutes of Health, Bethesda, MD, USA).

Reverse transcription-quantitative polymerase chain reaction $(R T-q P C R)$. Total RNA was extracted from cells using TRIzol ${ }^{\circledR}$ reagent (Invitrogen; Thermo Fisher Scientific, Inc.). The RNA was reverse transcribed using Moloney Murine Leukemia Virus RT (Invitrogen; Thermo Fisher Scientific, Inc.) according to the manufacturer's protocol. RT-qPCR was performed with the SYBR qPCR kit (Vazyme) using a Stratagene Mx3000 Quantitative RT-PCR System. Amplification procedures were as follows: $95^{\circ} \mathrm{C}$ for $5 \mathrm{~min}, 30$ cycles of $95^{\circ} \mathrm{C}$ for $20 \mathrm{sec}$, $62^{\circ} \mathrm{C}$ for $30 \mathrm{sec}$ and ultimately $72^{\circ} \mathrm{C}$ for $10 \mathrm{~min}$. The primer pairs for the human Akt1 gene were as follows: Akt1 forward 5'-GCAGCACGTGTACGAGAAGA-3'; Akt1 reverse 5'-GGT GTCAGTCTCCGACGTG-3'; GAPDH forward 5'-ATCAGC AATGCCTCCTGCAC-3'; GAPDH REVERSE 5'-TGGCAT GGACTGTGGTCATG-3'. The relative Akt1 mRNA level was normalized to GAPDH. Relative quantification was performed using the $2^{-\Delta \Delta \mathrm{Cq}}$ method (15).

Statistical analysis. All graphs were processed by GraphPad Prism version 5 (GraphPad Software, Inc., La Jolla, CA, USA). The data are displayed as the mean \pm standard deviation, and were analyzed using the SPSS 16.0 statistical package (SPSS, Inc., Chicago, IL, USA). Statistical significance among the indicated groups was determined by ANOVA following Tukey's test or unpaired Student's t-test. $\mathrm{P}<0.05$ was considered to indicate a statistically significance difference.

\section{Results}

Triptolide reduces the viability of C666-1 and NP69 cells. To investigate the cytotoxic properties of triptolide in the C666-1 and NP69 cell lines, cells were incubated with serial concentrations of triptolide (12.5, 25, 50 and $100 \mathrm{nM}$; Fig. 1A and B) for $24 \mathrm{~h}$. Significant differences were observed at $\geq 12.5 \mathrm{nM}$ in C666-1 cells, and at $100 \mathrm{nM}$ in NP69 cells. With increasing concentrations of triptolide, the viability of the C666-1 cells decreased in a dose-dependent manner. To study the association between cell viability and time, the C666-1 and NP69 cells were incubated with $50 \mathrm{nM}$ of triptolide and analyzed at 6,12 , 24 and 48 h (Fig. 1C and D). The viability of the C666-1 cells 

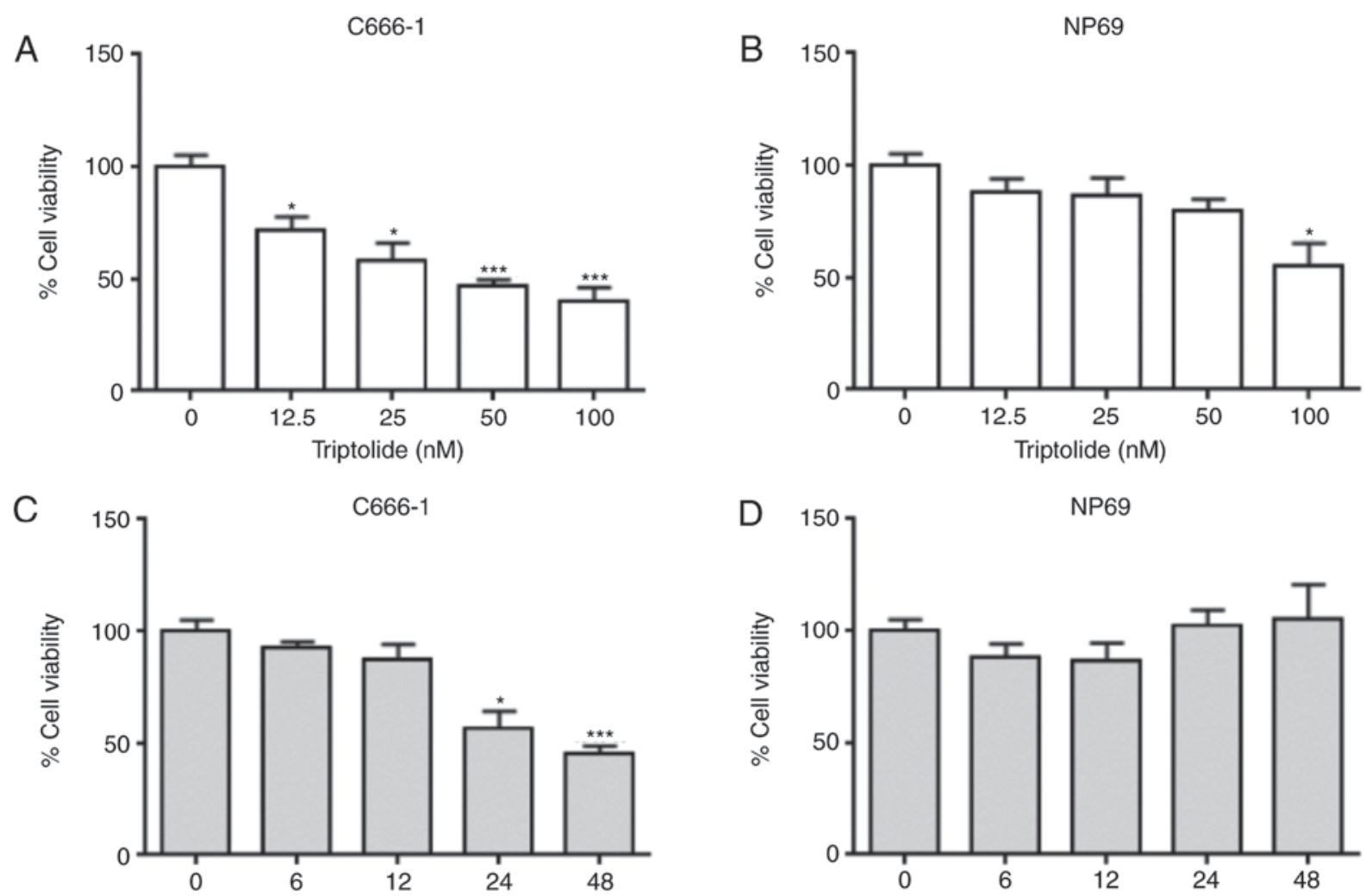

(h)

(h)

Figure 1. Triptolide reduces the viability of C666-1 and NP69 cells. The (A) C666-1 and (B) NP69 cells were treated with serial concentrations of triptolide and the cell viability was measured by MTT assay. The (C) C666-1 and (D) NP69 cells were respectively treated with $50 \mathrm{nM}$ triptolide and the viability was measured at $6,12,24$ and 48 h post induction. ${ }^{*} \mathrm{P}<0.05,{ }^{* * *} \mathrm{P}<0.01,{ }^{* * * *} \mathrm{P}<0.001$ vs. respective control.

was significantly reduced at $>12 \mathrm{~h}$, whilst no significant difference in viability was observed in NP69 cells at any of the time points tested. These results indicated that the cytotoxic effects of triptolide were more potent in C666-1 cells compared with NP69 cells.

Triptolide induces cell cycle arrest and promotes apoptosis. To investigate the role of triptolide-induced cytotoxicity on the cell cycle and apoptosis, cells were incubated at three concentrations of triptolide for $48 \mathrm{~h}$. The cell cycle assay suggested that treating C666-1 cells with 50 or $100 \mathrm{nM}$ triptolide caused an increase in the number of cells in the G0/G1 phase, and a decrease in the number of cells in the $\mathrm{G} 2 / \mathrm{M}$ phase, implying a G0/G1 arrest (Fig. 2A and B). Notably, although a reduction of the cell population in the $\mathrm{G} 2 / \mathrm{M}$ phase was also observed in NP69 cells, a greater number of cells were in the S phase rather than the G0/G1 phase. The flow cytometry assay demonstrated that cell apoptosis rates were increased in C666-1 cells in a dose-dependent manner, at concentrations of 12.5, 50 and $100 \mathrm{nM}$ triptolide. The NP69 cells displayed low levels of apoptosis (Fig. 2C and D).

Triptolide modulates pro-apoptotic protein expression and inhibits the PI3K/Akt signaling pathway. Given that triptolide displayed apoptotic activity in the C666-1 cells, the expression of cleaved caspase-3, Bax and Bcl-2 in cells treated with $50 \mathrm{nM}$ triptolide were measured by western blotting. Upregulated cleaved caspase- 3 and Bax expression, and downregulated $\mathrm{Bcl}-2$ expression, was observed in the
C666-1 cells (Fig. 3A and B). Previous studies have indicated that PI3K/Akt is involved in the anti-cancerous activity of triptolide, in addition to EBV-mediated carcinogenesis. The results of the western blotting confirmed that phosphorylation of Akt was significantly inhibited by triptolide in the C666-1 cell line (Fig. 3C-E). By contrast, no significant difference was observed in the expression levels of cleaved caspase-3, Bax, Bcl-2, p-Akt and Akt in NP69 cells following treatment with $50 \mathrm{nM}$ triptolide (Fig. 3F-J). In addition, the Akt1 mRNA expression level was significantly higher in C666-1 cells, which may indicate why C666-1 cells were more sensitive to triptolide compared with NP69 cells (Fig. 3K). These data implied that the pro-apoptotic effect and inhibition of proliferation induced by triptolide may be associated with the inhibition of the PI3K/Akt signaling pathway.

C666-1 cells pretreated with a PI3K/Akt inhibitor display impaired triptolide-induced apoptosis and inhibition of proliferation. To confirm the role of PI3K/Akt signaling in triptolide-induced cytotoxicity, C666-1 cells were pre-incubated with 1,10 and $50 \mu \mathrm{M}$ of the PI3K/Akt inhibitor LY294002 to block PI3K/Akt signaling. The C666-1 cells were subsequently incubated with $50 \mathrm{nM}$ triptolide; samples treated with LY294002 only served as a control. The results revealed that LY294002 inhibited the proliferation of the C666-1 cells in a dose-dependent manner, and with increasing concentrations of LY294002, the triptolide-induced inhibition of cell proliferation was further decreased (Fig. 4A and B). The expression levels of $\mathrm{p}$-Akt and the ratios of $\mathrm{p}$-Akt/Akt were significantly 
A

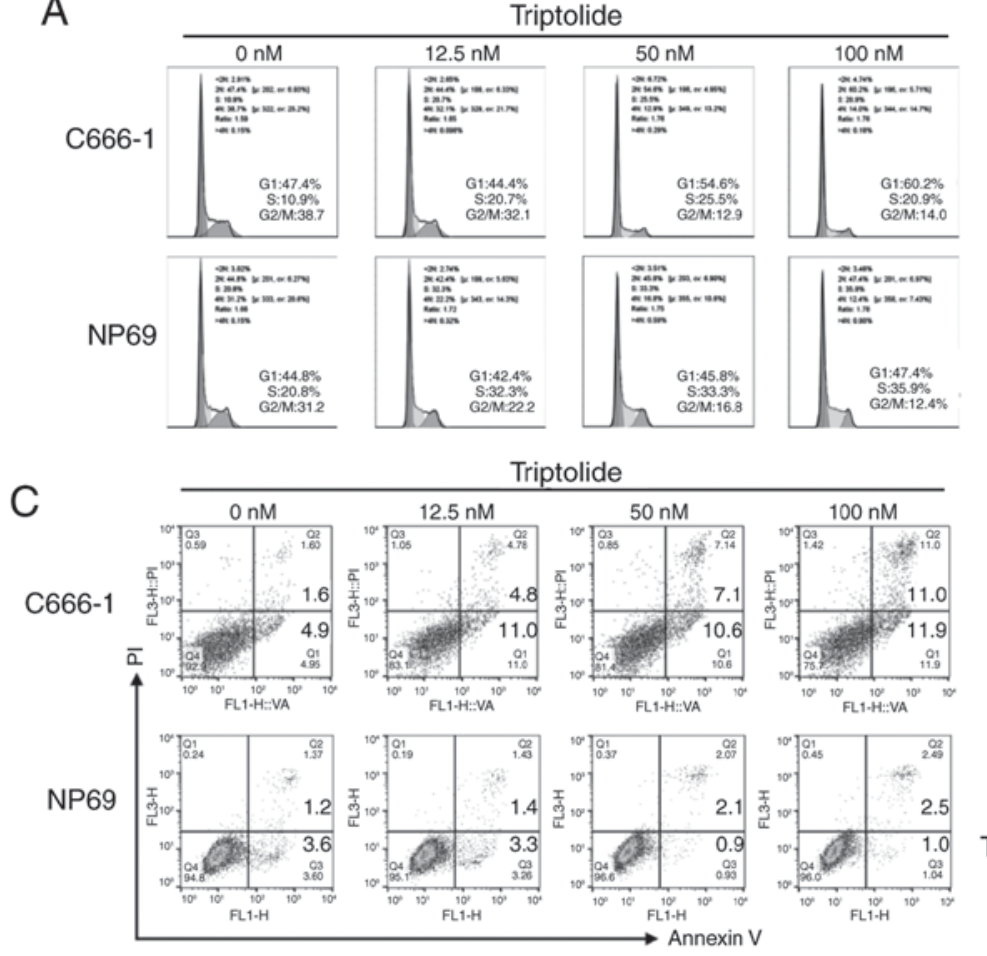

B
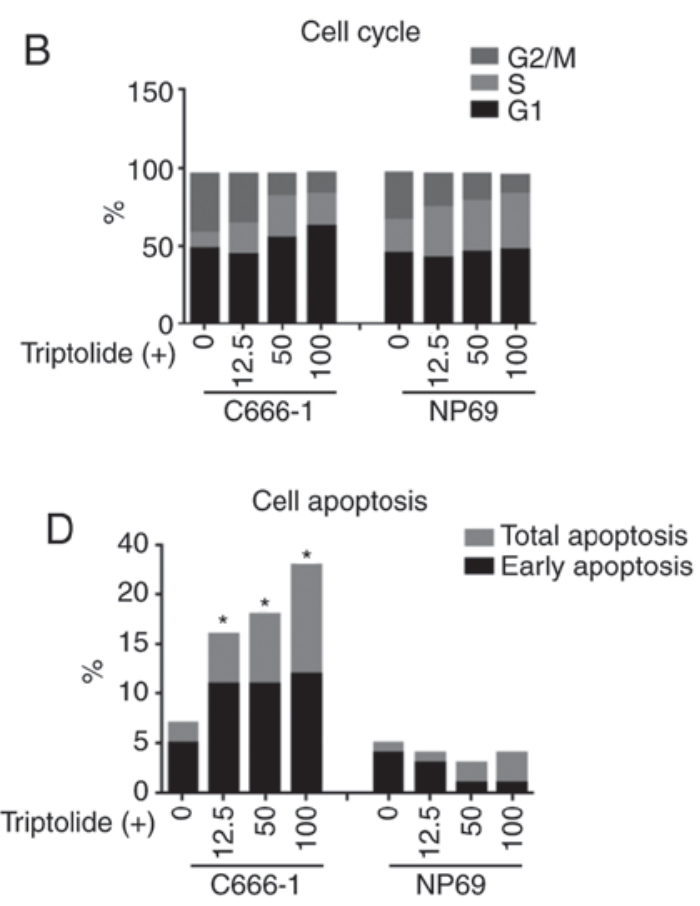

Figure 2. Triptolide induces cell cycle arrest and apoptosis in C666-1 and NP69 cells. The C666-1 and NP69 cells were treated with serial concentrations of triptolide for $48 \mathrm{~h}$, and the effects on the cell cycle were (A) measured by flow cytometry and (B) quantified. (C and D) The C666-1 and NP69 cells were treated with serial concentrations of triptolide and apoptosis was (C) measured by flow cytometry and (D) quantified. *P<0.05 vs. control. PI, propidium iodide.

reduced when treated with either LY294002 or triptolide (Fig. 4C and D). At a low concentration of LY294002, the addition of triptolide had a further inhibitory effect on the phosphorylation of Akt. FACS apoptosis assays were performed at $48 \mathrm{~h}$ post-addition of triptolide. The results indicated that the pro-apoptotic effect of LY294002 was dose-dependent, and a further increase in the number of apoptotic cells was observed with the addition of triptolide (Fig. 4E). The ratios of apoptotic cells are displayed in Fig. 4F. In summary, these results suggested that triptolide induces apoptosis and inhibits cell proliferation in a PI3K/Akt-dependent manner.

\section{Discussion}

The anti-cancer effects of triptolide have been discussed with respect to multiple types of cancer. However, the underlying mechanism and its application in NPC was yet to be explored. The results of the present study indicated that triptolide exerted greater toxicity in NPC C666-1 cells when compared with normal nasal epithelial NP69 cells. Triptolide inhibited proliferation and cell cycle progression, and induced apoptosis in C666-1 cells. The mechanism was associated with the activation of caspase-3, Bax, and the inhibition of $\mathrm{Bcl} 2$, in addition to Akt phosphorylation. The PI3K/Akt signaling pathway served an important role in the apoptosis and the inhibition of proliferation induced by triptolide.

Cell cycle arrest contributes to the inhibition of cancer cell proliferation induced by various cytotoxic anticancer agents. Triptolide may induce different types of cell cycle arrest, depending on the characteristics of the tumor cells concerned. A previous study indicated that triptolide results in the accumulation of certain cells in the $\mathrm{S}$ phase, including MDA-MB-231, HT-1080 and OS-RC-2 cells (15). In the present study, analysis of the cell cycle suggested that triptolide induced a potent G0/G1 phase arrest in NPC C666-1 cells and to some extent, influenced the accumulation of NP69 cells in the $\mathrm{S}$ phase.

Triptolide possesses potent anticancer activity with a half-maximal inhibitory concentration $\left(\mathrm{IC}_{50}\right)$ ranging between 4 and $50 \mathrm{nM}$ (16). Previous studies have identified that triptolide-associated apoptosis is the central cause of its cytotoxicity $(17,18)$. The underlying mechanism of apoptosis induced by triptolide is associated with the activation of caspase- 3,8 and 9, the cleavage of poly ADP ribose polymerase to release cytochrome $\mathrm{C}$ from mitochondria, and the initiation of cellular tumor antigen p53-dependent pathways (16). Of note, the Bcl-2 family dominates the intrinsic apoptosis pathways. The results of the present study suggested that triptolide promotes the activation of caspase-3, upregulates the pro-apoptotic protein Bax, and downregulates the anti-apoptotic protein $\mathrm{Bcl}-2$ to activate the mitochondrial apoptosis pathway.

Given that the PI3K/Akt signaling pathway is highlighted in EBV-positive NPC, the present study investigated any alterations in Akt signaling in C666-1 cells. The results indicated that Akt phosphorylation was significantly inhibited by triptolide. As Akt is identified as a key modulator of apoptosis and cell proliferation, C666-1 cells were pretreated with the PI3K/Akt inhibitor LY294002 prior to the assessment of proliferation and apoptosis. Increasing concentrations of LY294002 further induced triptolide-associated apoptosis and inhibition of cell proliferation in the C666-1 cells. In addition, studies have also demonstrated that the inactivation of Akt 
A

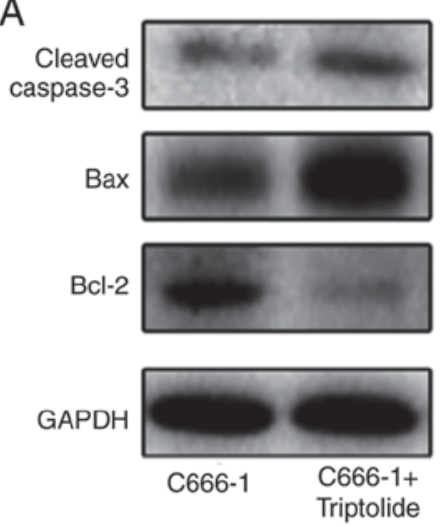

B

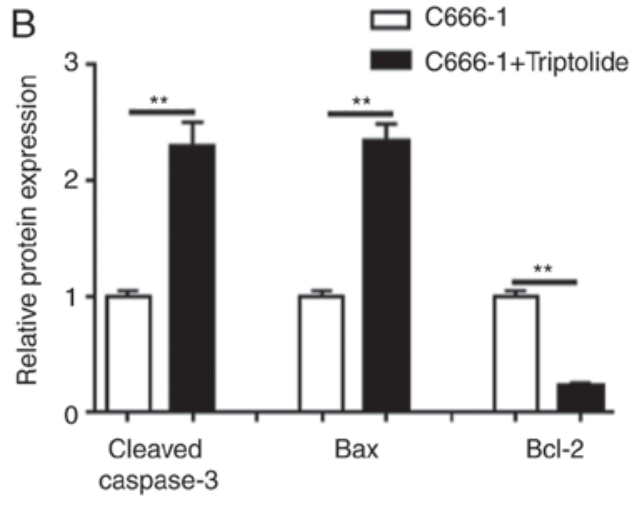

C

p-Akt

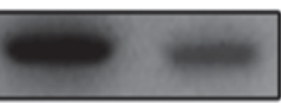

Akt

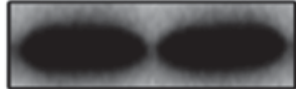

GAPDH

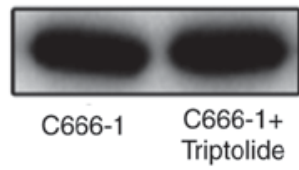

$\mathrm{F}$

Cleaved caspase-3

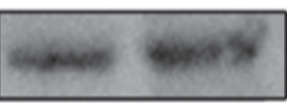

Bax

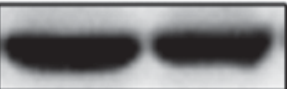

Bcl-2

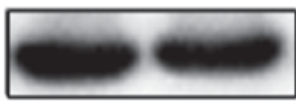

GAPDH

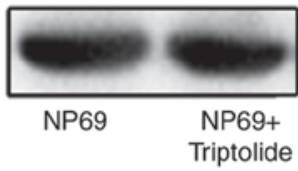

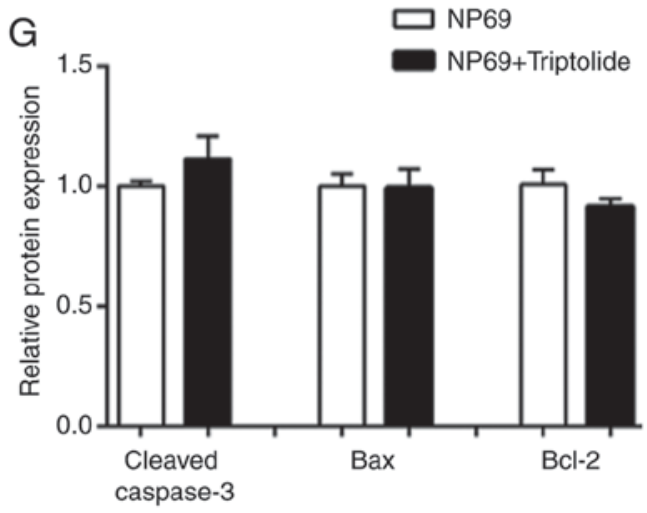

$\mathrm{H}$

p-Akt

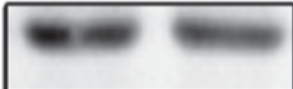

Akt

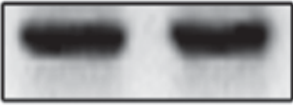

GAPDH
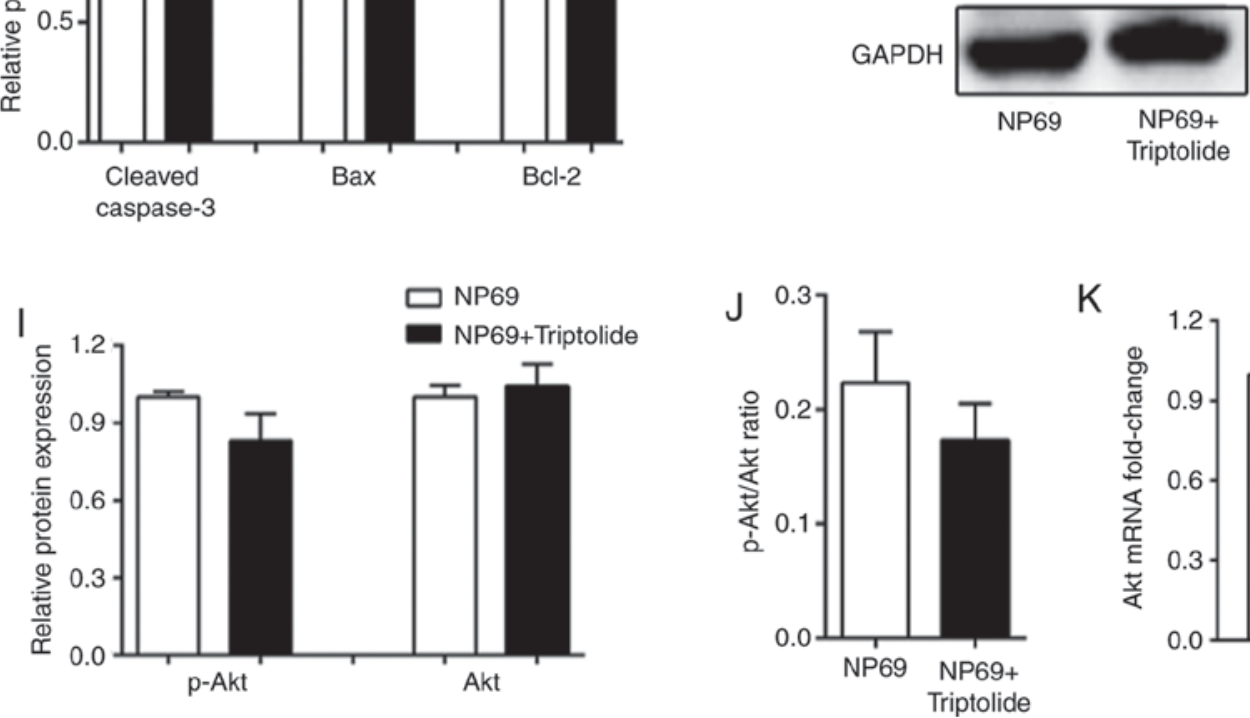

K

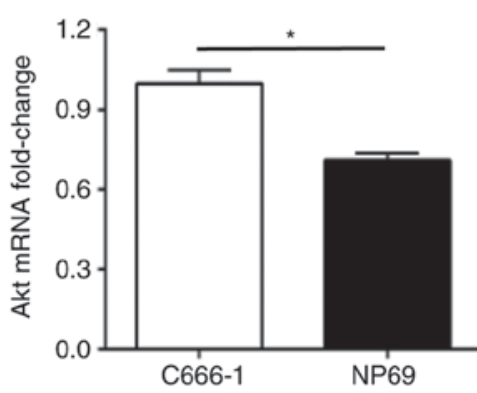

Figure 3. Triptolide modulates pro-apoptotic protein expression and inhibits the PI3K/Akt signaling pathway. C666-1 or NP69 cells were treated with 50 nM triptolide for $48 \mathrm{~h}$. (A) The protein expression levels of cleaved caspase-3, Bax and Bcl-2 were detected by western blotting. (B) The relative expression of cleaved caspase-3, Bax and Bcl-2 was normalized to GAPDH. (C) The protein expression levels of Akt and p-Akt were detected by western blotting. (D) The relative expression of Akt and p-Akt was normalized to GAPDH. (E) The p-Akt/Akt ratio was quantified. (F) The protein expression levels of cleaved caspase-3, Bax and Bcl-2 were detected by western blotting. (G) The relative expression of cleaved caspase-3, Bax and Bcl-2 was normalized to GAPDH. (H) The protein expression levels of Akt and p-Akt were detected by western blotting. (I) The relative expression of Akt and p-Akt was normalized to GAPDH. (J) The p-Akt/Akt ratio was quantified. (K) The Akt1 mRNA expression level was measured in the C666-1 and NP69 cells. * $\mathrm{P}<0.05$; ${ }^{* *} \mathrm{P}<0.01$. PI3K, phosphatidylinositol 3-kinase; Akt, RAC- $\alpha$ serine/threonine-protein kinase; p, phosphorylated; Bax, apoptosis regulator BAX; Bcl-2, apoptosis regulator Bcl-2. 

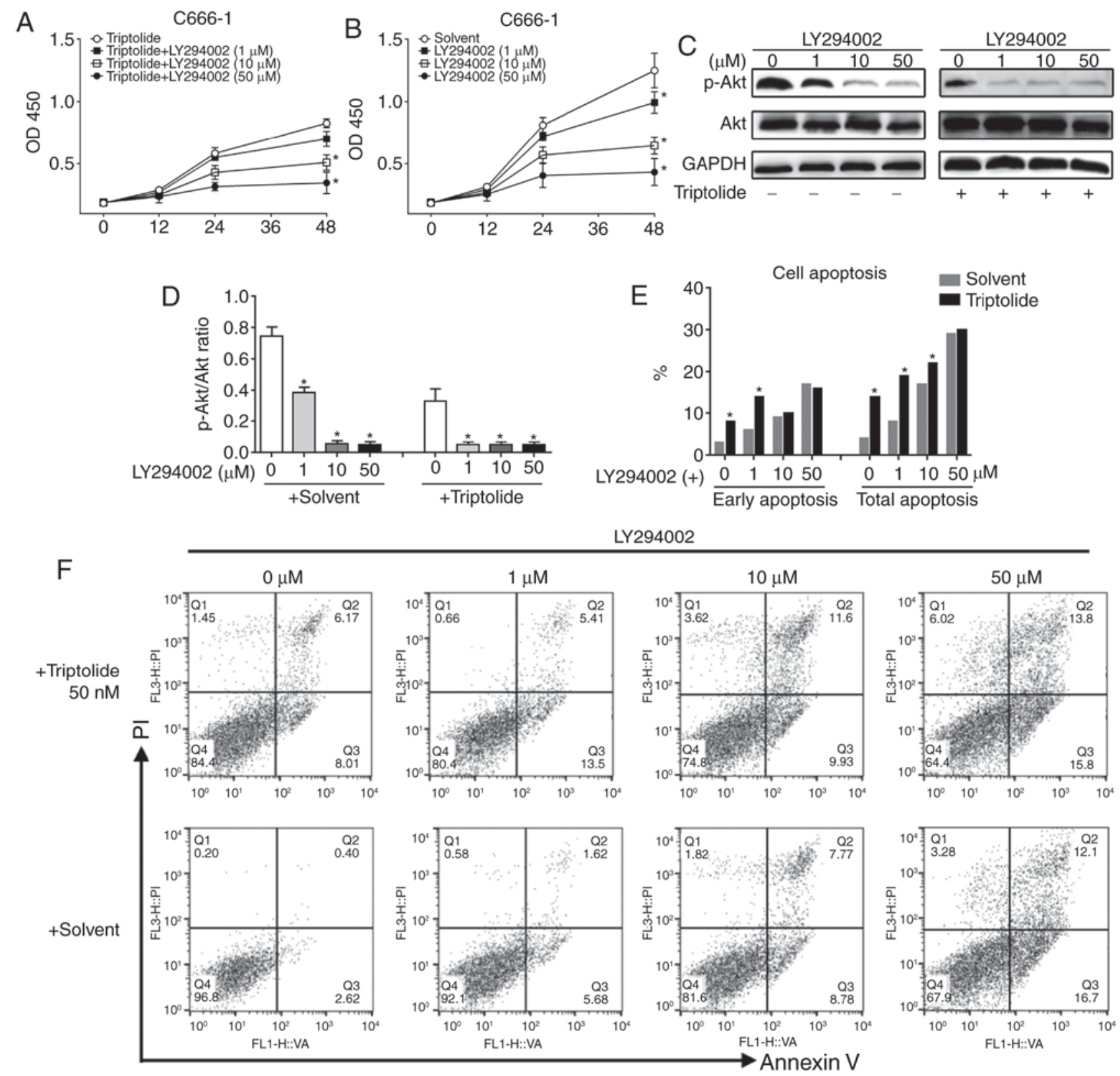

Figure 4. Pretreatment of C666-1 cells with the phosphatidylinositol 3-kinase/Akt inhibitor LY294002 counteracts the triptolide-induced inhibition of prolif-

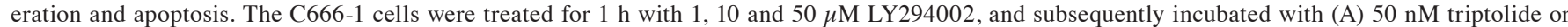
(B) control solvent. The cell viability was measured by MTT assay. The protein levels of p-Akt and Akt at $48 \mathrm{~h}$ were detected by (C) western blotting and (D) the p-Akt/Akt ratio was quantified. (E) Apoptosis was detected by flow cytometry and (F) quantified at $48 \mathrm{~h}$ post incubation with triptolide. "P $<0.05 \mathrm{vs}$. respective control. Akt, RAC- $\alpha$ serine/threonine-protein kinase; p, phosphorylated; PI, propidium iodide; OD, optical density.

is closely associated with the triptolide-induced inhibition of $\mathrm{N}$-terminally-truncated retinoid $\mathrm{X}$ receptor- $\alpha$ in various cancer cells, and E3 ubiquitin-protein ligase Mdm2 in human breast cancer $(15,19,20)$.

In conclusion, the present study demonstrated that triptolide reduced the viability of C666-1 cells. The NPC C666-1 cells were highly susceptible to triptolide and exhibited potent apoptosis following incubation in a PI3K/Akt-dependent manner.

\section{Acknowledgements}

The authors would like to acknowledge Mr. Shuihong Zhou, Dr Zhili Zhang and Mr. Libo Dai for their help with the design of the experiment and data analysis.

\section{Funding}

No funding was received.

\section{Availability of data and materials}

All data generated or analyzed during the present study are included in this published article.

\section{Authors' contributions}

MW and BC performed the experiments, analyzed the data and wrote the manuscript. LC, designed the experiment, drafted the manuscript, provided materials and supervised the experiments. 


\section{Ethics approval and consent to participate}

Not applicable.

\section{Patient consent for publication}

Not applicable.

\section{Competing interests}

The authors declare that they have no competing interests.

\section{References}

1. Lo KW, Chung GT and To KF: Deciphering the molecular genetic basis of NPC through molecular, cytogenetic, and epigenetic approaches. Semin Cancer Biol 22: 79-86, 2012.

2. Torre LA, Bray F, Siegel RL, Ferlay J, Lortet-Tieulent J and Jemal A Global cancer statistics, 2012. CA Cancer J Clin 65: 87-108, 2015.

3. Maxwell JH, Kumar B, Feng FY, McHugh JB, Cordell KG, Eisbruch A, Worden FP, Wolf GT, Prince ME, Moyer JS, et al: HPV-positive/p16-positive/EBV-negative nasopharyngeal carcinoma in white North Americans. Head Neck 32: 562-567, 2010.

4. Dawson CW, Port RJ and Young LS: The role of the EBV-encoded latent membrane proteins LMP1 and LMP2 in the pathogenesis of nasopharyngeal carcinoma (NPC). Semin Cancer Biol 22 144-153, 2012.

5. Shah KM, Stewart SE, Wei W, Woodman CB, O'Neil JD, Dawson CW and Young LS: The EBV-encoded latent membrane proteins, LMP2A and LMP2B, limit the actions of interferon by targeting interferon receptors for degradation. Oncogene 28: 3903-3914, 2009.

6. Dawson CW, Rickinson AB and Young LS: Epstein-barr virus latent membrane protein inhibits human epithelial cell differentiation. Nature 344: 777-780, 1990

7. Allen MD, Young LS and Dawson CW: The Epstein-barr virus-encoded LMP2A and LMP2B proteins promote epithelial cell spreading and motility. J Virol 79: 1789-1802, 2005.

8. Oda K, Okada J, Timmerman L, Rodriguez-Viciana P, Stokoe D Shoji K, Taketani Y, Kuramoto H, Knight ZA, Shokat KM and McCormick F: PIK3CA cooperates with other phosphatidylinositol 3'-kinase pathway mutations to effect oncogenic transformation. Cancer Res 68: 8127-8136, 2008.
9. Corson TW and Crews CM: Molecular understanding and modern application of traditional medicines: Triumphs and trials. Cell 130: 769-774, 2007.

10. Ma J, Dey M, Yang H, Poulev A, Pouleva R, Dorn R, Lipsky PE, Kennelly EJ and Raskin I: Anti-inflammatory and immunosuppressive compounds from Tripterygium wilfordii. Phytochemistry 68: 1172-1178, 2007.

11. Yang SX, Gao HL, Xie SS, Zhang WR and Long ZZ: Immunosuppression of triptolide and its effect on skin allograft survival. Int J Immunopharmacol 14: 963-969, 1992.

12. Banerjee S, Sangwan V,McGinn O, Chugh R, Dudeja V, Vickers SM and Saluja AK: Triptolide-induced cell death in pancreatic cancer is mediated by O-GlcNAc modification of transcription factor Sp1. J Biol Chem 288: 33927-33938, 2013.

13. Wu PP, Liu KC, Huang WW, Ma CY, Lin H, Yang JS and Chung JG: Triptolide induces apoptosis in human adrenal cancer NCI-H295 cells through a mitochondrial-dependent pathway. Oncol Rep 25: 551-557, 2011

14. Yang S, Chen J, Guo Z, Xu XM, Wang L, Pei XF, Yang J, Underhill CB and Zhang L: Triptolide inhibits the growth and metastasis of solid tumors. Mol Cancer Ther 2: 65-72, 2003.

15. Livak KJ and Schmittgen TD: Analysis of relative gene expression data using real-time quantitative PCR and the 2(-Delta Delta $\mathrm{C}(\mathrm{T}))$ method. Methods 25: 402-408, 2001.

16. Xiong J, Su T, Qu Z, Yang Q, Wang Y, Li J and Zhou S: Triptolide has anticancer and chemosensitization effects by down-regulating Akt activation through the MDM2/REST pathway in human breast cancer. Oncotarget 7: 23933-23946, 2016.

17. Chen Q, Lu Z, Jin Y, Wu Y and Pan J: Triptolide inhibits Jak2 transcription and induces apoptosis in human myeloproliferative disorder cells bearing Jak2V617F through caspase-3-mediated cleavage of Mcl-1. Cancer Lett 291: 246-255, 2010.

18. Carter BZ, Mak DH, Schober WD, Dietrich MF, Pinilla C, Vassilev LT, Reed JC and Andreeff M: Triptolide sensitizes AML cells to TRAIL-induced apoptosis via decrease of XIAP and p53-mediated increase of DR5. Blood 111: 3742-3750, 2008.

19. Liu L, Li G, Li Q, Jin Z, Zhang L, Zhou J, Hu X, Zhou T, Chen J and Gao N: Triptolide induces apoptosis in human leukemia cells through caspase-3-mediated ROCK1 activation and MLC phosphorylation. Cell Death Dis 4: e941, 2013.

20. Wang PY, Zeng WJ,Liu J, Wu YL, Ma Y, Zeng Z, Pang JY Zhang XK, Yan X, Wong AST and Zeng JZ: TRC4, an improved triptolide derivative, specifically targets to truncated form of retinoid X receptor-alpha in cancer cells. Biochemical pharmacology 124: 19-28, 2017. 